\title{
Epistemología y Ontología: un debate necesario para la Psicología hoy
}

\author{
Epistemology and Ontology: \\ a necessary debate for Psychology today
}

Fernando González Rey
Centro Universitario de Brasilia,
Brasil
Recibido: 22 de noviembre de 2008

Revisado: 22 de marzo de 2009

Aceptado: 9 de mayo de 2009

\section{Resumen}

En este artículo se discute el desarrollo de los aspectos ontológicos y epistemológicos del psicoanálisis, la psicología empírica apoyada en el comportamiento y la psicología soviética, que constituyeron tres representaciones ontológicas diferentes sobre la psique. Cada una de esas representaciones tuvo implicaciones epistemológicas y metodológicas diferentes. Especial atención se da a la psicología soviética, la cual, hasta hoy, continúa siendo bastante poco conocida en la psicología occidental. Esa psicología, tanto por las condiciones históricas en que emergió, como por las influencias filosóficas y culturales que recibió, tuvo importantes diferencias con los enfoques psicológicos más conocidos de aquel tiempo. A partir de ese marco, el autor presenta su propuesta de subjetividad en una perspectiva histórico-cultural, discutiendo las exigencias epistemológicas y metodológicas que la redefinición de ese término implica desde esta aproximación teórica.

Palabras clave: epistemología, subjetividad, ontología, teoría.

* Artículo de investigación documental.

** Correspondencia: Fernando González Rey, profesor investigador Centro Universitario de Brasilia, Brasil. Correo electrónico: gonzalezrey@terra.com.br. 


\section{Summary}

In this paper is discussed the development of the ontological and epistemological questions related to psychoanalysis, the empirical psychology supported on behavior and the soviet psychology. These approaches implied three different ontological representations on psyche. Each of those ontological representations was involved with different epistemological and methodological consequences. A special attention is given in the discussion to soviet psychology, an approach that up to now remains little known in the western psychology. Soviet psychology, either by the historical conditions of its emergence, or by received cultural and philosophical influences, had important differences with the more known psychological approaches in that time. Started from historical cultural approach, the author introduces the topic of the subjectivity from a cultural - historical standpoint, as a new ontological proposal. It is also discussed the epistemological and methodological demands resulted from the study of the subjectivity in this theoretical approach.

Key words: epistemology, subjectivity, ontology, theory.

\section{Introducción}

La psicología históricamente se preocupó poco de la discusión epistemológica, así como de la discusión de lo que entendía por psique. Un aspecto que influyó en esa tendencia fue la separación intencional que durante mucho tiempo fue asumida por la psicología en relación con la filosofía y con otras ciencias sociales. La idea de disciplina, apoyada en el concepto de objeto propuesto por Durkheim, fue asumida como un verdadero dogma por las diferentes tendencias de la psicología moderna, la que, con pocas excepciones, definió su objeto en procesos del individuo susceptibles de leyes propias, ya sea a nivel intrapsíquico o comportamental. De esas tendencias emergieron dos de las teorías más significativas del desarrollo de la psicología; el behaviorismo y el psicoanálisis.

La ausencia de discusión sobre las cuestiones epistemológicas llevó a la psicología a una definición positivista de ciencia, con sus consecuencias en términos de una comprensión del saber objetiva, instrumental y a-teóricamente, lo que se evidenció en el carácter experimental y cuantitativo de su metodología dominante. De hecho, algunas de las tendencias más importantes del saber psicológico quedaron excluidas de la definición de ciencia, por no adaptarse a los cánones de cientificidad definidos por el positivismo.
La emergencia de la discusión posmoderna en la filosofía y en el resto de las ciencias sociales, acentúo el énfasis de las cuestiones epistemológicas al interior de la psicología. De una forma u otra, los nuevos focos de discusión que se extendieron con una fuerza y rapidez inéditas al campo de las ciencias sociales en general, produjeron un rechazo al positivismo: ¡Nadie quería ser positivista!

Sin embargo, los acuciantes temas que la discusión posmoderna trajo al campo de la psicología, sólo fueron abiertamente discutidos en sus implicaciones para esta área por un número reducido de autores, a pesar de lo cual dichas discusiones tuvieron un fuerte impacto. Las nuevas tendencias teóricas que asumían de forma explícita las posiciones posmodernas, como el construccionismo social y las diferentes versiones de constructivismo pos piagetano, tuvieron un fuerte impacto en la agenda crítica que aceleradamente invadió la psicología desde finales de la década de los años setenta del siglo XX. Esa agenda crítica se extendió prácticamente a todas las áreas de la psicología; sin embargo, muchas de las publicaciones más relevantes de la psicología y de las instituciones de su enseñanza, parecen no haberse enterado de dichos eventos.

Los "nuevos aires" en el pensamiento psicológico irrumpieron con tal fuerza el escenario de la psicología que, en algunos casos, proclamaron el 
fin de la epistemología, la obsolescencia de la ontología y la muerte del sujeto y de la historia, actuando en esa crítica como si esos conceptos tuvieran un valor intrínseco en sí mismos, y no fueran parte de sistemas teóricos dentro de los cuales asumen su significado. La crítica radicalmente antimetafísica definía metafísicamente su propio objeto de crítica. Los psicólogos que nunca habían discutido activamente las cuestiones epistemológicas y ontológicas de la psicología, de pronto aparecían excluyéndolas de sus agendas por su carácter metafísico. El nuevo pensamiento, que se orientaba contra el dogma, aparecía como dogma, acompañado del peligro que toda "moda dominante" trae, la banalización, la superficialidad y la repetición mimética de afirmaciones generales, más como ritual de la moda, que como necesidad real de la construcción del conocimiento.

En este artículo, por consiguiente, pretendo discutir las dos cuestiones anunciadas en el título y fundamentar la importancia que les veo para el desarrollo actual de la psicología. Unido a esto haré explícitas mis posiciones a partir de mi propuesta sobre la subjetividad en un marco histórico-cultural.

\section{El desarrollo epistemológico y las diferentes ontologías de la psicología}

Las diferentes tendencias del pensamiento psicológico moderno discutieron poco las implicaciones epistemológicas asociadas con sus formas particulares de producción de conocimiento, así como sus definiciones de psique. El behaviorismo, de manera general, se presentó como la tendencia científica de la psicología moderna apoyada en su carácter experimental y otorgó como lugar central la demostración empírica como criterio de legitimidad del saber producido. Esa orientación epistemológica se adecuó perfectamente a la definición del comportamiento como objeto de estudio de la psicología.

El modelo hipotético deductivo no se desarrolló sólo desde una perspectiva experimental, sino también, desde una perspectiva centrada en la medición apoyada en cuestionarios, tests, y otros instrumentos susceptibles a la cuantificación de sus resultados. Al igual que la orientación experimental, esa orientación cuantitativa apoyada en instrumentos de medición, se apoyó en una representación empírica de ciencia basada en la producción de datos. Los datos encontraban su significado en términos de los instrumentos que los medían y de las estadísticas que los correlacionaban. Con la estadística, el principio de la inducción asumía sofisticadas formas de expresión en la psicología.

La psicología que se apoyó en el positivismo, buscó en la evidencia (ya fuera inmediata y fáctica en el experimento, o mediata a través de la estadística), el criterio fundamental de legitimidad científica y de objetividad. La demostración y los resultados cuantificados pasaron a ser el criterio de cientificidad del conocimiento y aparecieron como garantía de la objetividad del conocimiento. La definición de lo empírico apareció como contraposición a lo teórico y abrió una falsa división entre ciencia y filosofía; la ciencia se definía como saber objetivo instrumental y la filosofía como saber teórico - especulativo.

La mayor parte de la investigación desarrollada con esa orientación instrumental cuantitativa se apoyaba en una ontología comportamental; tanto el behaviorismo, como las investigaciones empíricas con muestras grandes, apoyadas en la medición de variables, no reconocían ninguna especificidad en las variables que usaban; todas estaban referidas al comportamiento. No existía discusión teórica sobre la naturaleza de los procesos medidos; se media con base en definiciones operacionales. Esa forma de producir conocimiento dejaba fuera todas las cuestiones que no se adecuaran a ese modelo de trabajo metodológico, excluyéndose del quehacer científico el papel de las ideas y de las teorías en la producción del conocimiento.

La pregunta sobre qué estudiar es fundamental y en su respuesta está contenida la dimensión ontológica del conocimiento. Todo problema a ser estudiado representa una construcción teórica, por lo que, en ningún caso, podría verse como 
una evidencia empírica. Esta cuestión tan simple es todavía hoy motivo de discusión entre los psicólogos, pues el concepto de ciencia empírica se acompañó de la ilusión al representar en lo empírico la expresión directa de la realidad, independiente de las representaciones del investigador. Los límites que ya Kant había definido a la razón en el conocimiento resultaban totalmente ignorados. Por detrás de esta ilusión los conceptos no aparecían como construcciones intelectuales, sino como el significado de los datos. Los conceptos se naturalizaban como realidades.

La idea de definición operacional obligaba a transformar lo psíquico en dimensiones concretas. La timidez aparecía como conjunto de comportamientos universales asociados a esa categoría, ante lo cual cabe preguntarse, ¿será que el tímido no puede expresarse a través de comportamientos diferentes?, ¿será que un atributo psíquico se sustancializa en comportamientos concretos? Esta discusión realmente no se realizaba, pues era algo asumido por quienes compartían ese marco teórico; la evidencia comportamental es el único elemento objetivo para estudiar la psique.

Freud, a diferencia de la tendencia instrumentalinductiva de base comportamental, se orientó por otra estrategia metodológica. Al definir la génesis universal del inconsciente y la personalidad intrapsíquica, Freud entró en contradicción con los recursos epistemológicos de que disponía para generar evidencias sobre ese carácter universal. Al hacer eso extrapoló el valor heurístico de su teoría, intentando explicar todas las dinámicas de la psique a partir de una misma génesis.

La interpretación apareció como necesidad del estudio del inconsciente, pero su forma de uso (más que una expresión hermenéutica), pasó a ser un camino de descubrimiento de los procesos más profundos de la psique. Para Freud el objeto del psicoanálisis fue el inconsciente, su dinámica y sus procesos, pero nunca definió la naturaleza cualitativa diferenciada de esos procesos. Al asumir que el inconsciente se organizaba sobre la base de tendencias reprimidas cargadas de libido -de hecho- Freud lo definió en términos fisicalistas, como energía. Esa representación estuvo estrechamente asociada con la reificación de la sexualidad como base de toda la motivación humana; el deseo sexual, en su obra, aparece como desdoblamiento de una pulsión biológicamente definida. Sobre la base del deseo es que se definen las fuerzas y conflictos sobre las que se organiza la personalidad intrapsíquica, que Freud definió como segunda tópica; la energía asociada a un deseo universal fue la base dinámica del inconsciente.

En Freud no aparece una definición ontológica diferenciada del deseo en relación con la pulsión; aquel es un desdoblamiento de la pulsión. Su representación de psique se mantuvo apoyada por un referente objetivo; el deseo no cambia su naturaleza, permanece de forma universal asociado a la sexualidad. Esa forma de pensar el deseo lo define sobre una base objetiva: lo subjetivo sólo aparece en las formas de expresión y satisfacción del deseo, pero no en el deseo mismo. Es por esta razón que no podemos afirmar que Freud introdujera una nueva definición ontológica sobre la psique: la psique, en su génesis y desarrollo, continúa siendo una expresión de un deseo universal de base orgánica.

La subjetividad es una definición ontológica, no una referencia a una condición particular de un proceso psíquico, como pueden ser su carácter intrapsíquico, íntimo, inconsciente, etc. Al asumir el inconsciente como su objeto de interés, Freud creó una realidad que se organiza más allá del comportamiento y que no aparece de forma directa en él, siendo sus vías de acceso indirectas por definición: síntomas, sueños y actos fallidos. Ante su definición de inconsciente Freud creó un método de trabajo con principios epistemológicos implícitos que representaron una alternativa a la orientación cuantitativa-experimental.

Frente a estas consideraciones ¿qué aspectos epistemológicos diferencian la aproximación psicoanalítica y qué consecuencias tuvieron para el desarrollo de la psicología? Creo que las implicaciones epistemológicas del psicoanálisis pasaron desapercibidas para el propio Freud y para la mayoría de los psicoanalistas, para quienes, debido, en parte, al propio clima epistemológico de la 
época, el psicoanálisis se fue convirtiendo en verdad incuestionable. La idea de inconsciente, en lugar de ser considerada como una herramienta de inteligibilidad, se transformó en realidad y su conocimiento a, través del psicoanálisis, en verdad. Más allá de la conciencia epistemológica del propio psicoanálisis, su influencia en la reivindicación de la clínica como espacio de producción de conocimiento, así como su reivindicación de la importancia de la relación para el conocimiento psicológico, hizo de la clínica un espacio de saber que, directa o indirectamente, llevó a cuestionar muchos de los principios dominantes del cientificismo positivista en la psicología (González Rey, 2002).

Sin embargo, el psicoanálisis se mantuvo mucho más centrado en procesos asociados al ejercicio de la clínica que en la investigación científica, lo que se hizo evidente de forma explícita en el propio cuestionamiento de Lacan sobre su status científico, cuestionamiento apoyado también por Foucault en aquel momento. El rechazo al status de ciencia del psicoanálisis, defendido por Lacan y Foucault, se apoyó en el concepto de ciencia empírica, lo que de cierta manera legitimaba que esa fuera la única forma de hacer ciencia. Ese rechazo tuvo implicaciones para el propio desarrollo del psicoanálisis, pues no se preocupó por el desarrollo de una metodología de investigación que respondiera a las necesidades derivadas de la especificidad de sus definiciones teóricas. El psicoanálisis no consiguió desarrollar los aspectos metodológicos que le permitieran un diálogo con lo empírico.

A pesar del carácter metafísico y determinista de sus construcciones teóricas, el psicoanálisis otorgó a la construcción teórica y a la interpretación un valor esencial, lo que le permitió el reconocimiento de lo singular como fuente esencial de producción de conocimiento. Esos hechos alimentaron un imaginario epistemológico diferente, pero que no se defendió explícitamente como alternativa de una nueva forma de investigación, lo que impidió una expresión metodológica que le permitiera mantener viva la tensión entre lo teórico y lo empírico. El momento empírico, en última instancia, era significado desde anticipa- ciones teóricas formuladas a priori sobre los principios y categorías más generales de la teoría, se imponían sin especificar su viabilidad real en la información empírica.

El juego entre lo teórico y lo empírico, mediado por las limitaciones necesarias que toda construcción implica, fue desconsiderado por el psicoanálisis de forma general, lo que condujo a la reificación de la teoría. La teoría se dogmatizó, perdió su capacidad de crecimiento y terminó como un conjunto de significados de carácter metafísico. La psique se erigió como universal y fundacional en relación con la cultura y la historia. Esa tendencia se expresa de múltiples formas en diferentes autores del psicoanálisis. Así, por ejemplo Jones afirmó (1920):

En cuanto a las influencias culturales ellas también son el producto de motivos biológicos; así, aquellas nunca están un paso más allá de éstas. Vea, por ejemplo, el Complejo de Edipo, que consideramos muy fundamental y, hasta posiblemente innato... ignoramos exactamente cómo, pero, sea como sea, es una tendencia fundamental. Muy bien, veamos ahora lo que pasa en una determinada sociedad, digamos en la sociedad alemana, donde el padre es mucho más importante, promulga leyes, etc. Es claro que usted esperaría encontrar ahí el Complejo de Edipo. Pero, y las otras sociedades donde el padre no tiene tanta importancia... Bien, llamarán a eso influencia ambiental o cultural. Es correcto, pueden llamar así; pero eso causa naturalmente un cambio en la forma adoptada por las reacciones biológicas, es como una presión (p. 149).

Se percibe en la cita el peso que atribuye el autor a lo biológico, en ese caso, identificándolo con lo que pertenece a las tendencias y pulsiones universales de la persona. La cultura aparece reducida a una presión, algo externo, algo que cambia sólo la expresión de las tendencias universales de la persona, que son precisamente aquéllas sobre las que se erige la teoría psicoanalítica. Ese pensamiento de Jones (1920) permite percibir claramente una de las limitaciones mayores de 
la representación teórica propuesta por Freud; el carácter externo de la cultura en relación con la psique, la que está esencialmente apoyada en tendencias biosomáticas inherentes a la persona.

Desde el punto de vista ontológico, lo anterior no permite especificar el carácter particular de la psique; ella es un resultado, no tiene carácter generador, es un resultado de un conflicto universal de fuerzas presentes en la propia estructura intrapsíquica. Esa estructura podrá cambiar sus dinámicas y formas de expresión, pero nunca sus componentes y conflictos, que están por encima de la cultura y de la historia. Esa definición ontológica dificulta definir al psicoanálisis como hermenéutica, en el sentido en que usaron el término, tanto Heidegger como Gadamer; para ambos, y de forma más acabada para Gadamer, el saber hermenéutico no está orientado ni a la verificación ni al descubrimiento, manteniéndose como proceso en desarrollo, susceptible de formas nuevas en contextos diferentes. La hermenéutica en la tradición moderna -asociada estrechamente a los autores anteriores-, representó una ruptura con la substancialización de los significados fuera del contexto teórico en que fueron producidos. En este sentido, Como nos recuerda Gadamer (2007):

Si continuó hablando de "conciencia", eso no es ninguna confesión de adhesión ni a Aristóteles, ni a Hegel. Lo que está en cuestión aquí es saber que la conciencia no es ninguna res (cosa en griego)... La meta de la reconstrucción es dejar al concepto hablar una vez más en su relación con la lengua viva. Esa es una tarea hermenéutica. Ella no tiene nada en común con un discurso oscuro sobre el origen y lo originario (p. 95).

La hermeneútica va exactamente en el sentido contrario de la vocación de Freud por los orígenes y causas últimas del comportamiento humano. Sin embargo, el psicoanálisis no termina en Freud, a pesar de que su imaginario permanece unido al espíritu de su teoría en muchos aspectos. En una afirmación realmente hermenéutica, y de profundo carácter antimetafísico, Roudinesco (1995) sostiene:
Todos los estudios de casos son construidos como "ficciones necesarias" (La autora refiere a Merleau Ponty (1991) el origen de este término en nota al pie) a la legitimación de las hipótesis del autor. El caso solamente posee valor de verdad porque es escrito como ficción. Generalmente es adaptado a la nosografía de la época en que fue escrito. En otras palabras, Anna. O, el caso princeps de la histeria vienense de finales del siglo XIX, hoy en día no sería más considerada una histérica, pues el concepto de histeria mudó mucho desde la emergencia del saber psicoanalítico" (pp. 98).

Las categorías concretas nunca pueden tener un valor universal, como intenta defender Jones en la cita anterior; un concepto es siempre una producción intelectual asociada a cierta inteligibilidad, cuyo significado siempre está contextualizado en la temporalidad de un sistema teórico. Ese relativismo no es un agnosticismo, es la condición del saber humano, su carácter limitado y parcial en relación con la realidad que estudia. Sin embargo, yo defiendo la legitimidad del saber como forma de inteligibilidad; la inteligibilidad es la relación posible entre realidad y saber, asociada a opciones de modificación sobre la realidad a partir de ese saber. Las “demostraciones" son momentos de convergencia entre ciertas prácticas humanas y la realidad; ellas generan inteligibilidad en ese momento, abren caminos de nuevas acciones y nuevos saberes, pero no son nunca una evidencia final de un estado de la realidad.

Las "demostraciones" sólo son posibles en los marcos de una teoría, no en el sistema de la realidad. Las teorías son construcciones intelectuales capaces de crecer y desarrollar prácticas específicas sobre formas que toma la realidad en términos de la teoría; las teorías son accesos a la realidad en términos del saber, pero nunca representan un saber sobre el "ser" fuera de los recursos de inteligibilidad de la teoría.

Los modelos de pensamiento nunca pueden identificarse como sinónimos de la realidad que aparece en ellos. Como críticamente expresa Ponty (2006) en relación con la dialéctica de la naturaleza propuesta por Engels, la naturaleza no es 
dialéctica, es una forma de significar aspectos de la realidad. Es precisamente sobre ese aspecto definitorio del saber humano que introduje la categoría de zona de sentido (González Rey, 1996) para destacar que, lo más perdurable de un saber son los espacios de inteligibilidad que abre sobre un problema; la idea de inconsciente se perpetuó y es parte de una multiplicidad de representaciones sobre la psique humana; sin embargo, mostró que es mucho más compleja y disímil que las categorías usadas por Freud para dar cuenta de ese fenómeno. Es imposible que los procesos inconscientes, aceptados hoy por la mayoría de las representaciones teóricas sobre la psique humana, sean atrapados en su completitud por alguna teoría, simplemente porque las categorías son producciones humanas de inteligibilidad y no una expresión concreta de los procesos que ellas significan.

Me he detenido en dos sistemas generales que asumen dos ontologías diferentes que, a su vez, conducen a representaciones epistemológicas distintas; una asociada a una visión universal de metodología (el behaviorismo) que ha sido criticada por Danziger (1990) como metodolatría y por Koch (1981) como fetichismo metodológico; otra, asociada al psicoanálisis que, sin conciencia metodológica, no consiguió afirmarse dentro de una visión de ciencia, como lo hizo en una visión de clínica, convirtiéndose en el modelo de pensamiento más influyente de las teorías psicológicas basadas en la clínica. Esa división entre ciencia y clínica ha permitido que la representación dominante sobre la investigación científica en psicología se asocie al carácter inductivo, descriptivo e instrumental de la ciencia empírica, dejando la teoría asociada a la metafísica, la especulación y el hermetismo conceptual.

Este recorrido onto-epistemológico, que no es frecuente en los textos de psicología, debido, entre otras cosas, a la naturalización de las teorías como verdades, no pretende explorar todas las tendencias de la psicología sino aquellas que, en mi opinión, han sido más influyentes y abarcadoras en esta disciplina. En ese sentido, he decidido terminar este tópico con una tendencia que viene ganando espacio creciente en el mundo: me refiero a la psicología histórico-cultural.

\section{Relevancia de la psicología soviética en sus avances ontológico - epistemológicos}

La psicología soviética representó un movimiento sui géneris en la psicología moderna por diversas razones:

- Asumió de forma explícita una filosofía como la base de sus construcciones, pues esa filosofía no había sido relevante a ninguna de las teorías psicológicas del periodo moderno: el marxismo.

- En la forma en que los pioneros de la psicología soviética (Lazursky, Kornilov, Basov, Vygotsky, Rubinstein, Ananiev, entre otros) asumieron el marxismo, la dialéctica (como modelo de pensamiento) tuvo una particular influencia, lo que les facilitó la representación de la psique como sistema en movimiento, en desarrollo, estimulando una sensibilidad de interdisciplinaridad con otras ciencias sociales y con la filosofía, aspectos ausentes de forma general en la psicología moderna.

- El vínculo explícito con la filosofía facilitó el desarrollo de una sólida orientación teórica, apareciendo una preocupación explícita, tanto por las cuestiones ontológicas como metodológicas. En realidad, como veremos más adelante, esa preocupación metodológica tenía un carácter epistemológico, solo que la extrañeza del término entre los psicólogos, también impidió que se asumiera de forma explícita en aquella psicología.

Las razones argüidas hicieron de la psicología soviética un campo fecundo de aportes que no han sido plenamente asumidos hasta hoy por la psicología occidental. A ese hecho se unen los prejuicios ideológicos que engendró, y que pasaron a ser parte, hasta hoy, de la subjetividad social occidental, aparte de las dificultades del propio idioma ruso. La psicología soviética ha aparecido en occidente esencialmente a través de una de sus figuras esenciales, Vygotsky, cuyo pensamiento, con gran frecuencia, al ser separado de aquel movimiento más general de la psicología y 
descontextualizado del momento histórico en que realizó su obra, ha sido simplificado y banalizado.

La psicología soviética, como toda teoría psicológica, estuvo sometida no sólo a sus contradicciones internas derivadas de las diferentes tendencias de sus más influyentes figuras, sino también a presiones y contradicciones externas, resultantes de un contexto definido por una de las revoluciones más impactantes de la historia de la humanidad. Esas presiones, en especial las que comenzaron a aparecer en el momento institucional - conservador de aquella revolución, impactaron directamente en el desarrollo de aquella psicología, en el que la vocación dialéctica de algunos de sus fundadores se vio limitada por las circunstancias, y no sólo por el desarrollo de su propio pensamiento. Quizás el principal impacto de aquel momento lo representó el proceso de ideologización y dogmatización creciente de ese período histórico, lo que determinó presiones políticas, sociales e institucionales fuertes para los fundadores de la psicología soviética.

Particularmente fueron Vygotsky y Rubinstein los autores que más se interesaron por las cuestiones teóricas y metodológicas generales de la psicología. Entre ellos existieron puntos de vista diferentes; sin embargo, por lo que parece, en las referencias que he encontrado de Rubinstein sobre Vygotsky, existió una relación de respeto. Por el contrario, las relaciones de Rubinstein y Leontiev fueron mucho más tensas y contradictorias, representando quizás el conflicto más fuerte de la historia de la psicología soviética, después de los conflictos iniciales entre Kornilov y Chelpanov, que marcaron el inicio de la orientación marxista de aquella psicología. Los objetivos del presente artículo no me permiten extenderme en los aspectos históricos de aquella psicología, sino ceñirme a una pequeña ubicación de aquel contexto para los lectores no familiarizados con él ${ }^{1}$, dado que fue este movimiento el que generó un enfoque histórico-cultural en la psicología. A pesar de las diferencias entre Vygotsky y Rubinstein, ambos tenían muchos aspectos en común

1 Para mayor información sobre la psicología soviética ver González Rey "El Pensamiento de Vygotsky: desdoblamientos, contradicciones y desarrollos" (en proceso de edición. Editorial Trillas, 2008). en su representación general sobre la psicología. Para ambos la psique era inseparable de la acción, aspectos que se mantuvieron separados en las principales tendencias modernas de la psicología. Los dos se preocuparon por una representación de psique como sistema, en el que los aspectos cognitivos y afectivos aparecen integrados y en desarrollo. En el plano metodológico, tanto uno como otro, explicitaron las necesidades derivadas de sus definiciones teóricas por lo cual surge el siguiente interrogante ¿Cuáles fueron las principales contribuciones de esa psicología en los términos ontoepistemológicos que estamos analizando?

- En primer lugar su preocupación por el reconocimiento de la especificidad ontológica de la psique. Para ambos la psique humana era cualitativamente diferente de la animal, y se especificaba en relación con los procesos que participaban de su génesis. En este sentido Rubinstein escribió (1964):

A un mismo significado fisiológico de leyes variables, que figuran en las fórmulas fisiológicas, corresponde siempre una verdadera escala de significados psicológicos distintos. Los fenómenos psíquicos, por ende, sin dejar de formar parte inseparable de los fenómenos fisiológicos se diferencian de ellos (p.40).

- En segundo lugar, para ambos las funciones psíquicas expresan momentos del funcionamiento psíquico en general. Es imposible aislar la acción o los procesos psíquicos, de la psique como sistema. La idea de sistema, tanto Vygotsky como Rubinstein, se la representaron ora en la personalidad, ora en la conciencia, o en la espiritualidad. Sin embargo, ninguno de ellos llegó a definirla en términos de su naturaleza subjetiva, aunque ambos se aproximaron a esa idea en algunos momentos de su obra, mientras en otros se alejaron de ella.

El camino de definiciones ontológicas de la psicología pasó, tanto en Vygotsky como en Rubisntein, por un conjunto de problemas y contradicciones que, en alguna medida, expresaron la presión de 
tipo objetivo que la definición leninista de reflejo implicó para la comprensión de la psique. La idea de reflejo impedía el desarrollo del tema de la subjetividad, pues implicaba comprender la psique como reproducción de la realidad: ese principio generó en el imaginario de la psicología histórico-cultural, de forma general, la idea de un determinismo externo o sociologista, para el cual la psique aparece como efecto, como consecuencia, y no como producción.

De cualquier forma, la tendencia a representarse la psique como sistema que está más allá de las evidencias comportamentales, implicó un conjunto de consecuencias metodológicas que, como comentamos antes al analizar el psicoanálisis de Freud, tuvieron importantes consecuencias epistemológicas implícitas. A diferencia del psicoanálisis, los autores de la psicología soviética abordaron esa discusión con conciencia metodológica.

Por primera vez aparece en la psicología una orientación interpretativa inseparable de lo empírico. Vygotsky primero, y después Rubinstein, asumen una posición interpretativa ante el saber. Rubinstein escribe (1964):

Los testimonios de la conciencia y los "datos inmediatos" de la vivencia, para su conocimiento auténtico, han de someterse a una interpretación como si se tratara del texto de un discurso ${ }^{2}$. Para comprender un discurso no como objeto de ejercicios gramaticales, sino como un hecho vital en su auténtico significado, para comprender al hablante y no solo el texto formal de su discurso, es necesario descifrar, tras el texto, su "subtexto", poniendo de relieve no sólo lo que el hombre ha dicho formalmente, sino, además, lo que deseaba o tenía la intención de decir, o sea, el motivo y el fin de su discurso, determinantes del sentido interior del mismo (p. 229).

2 Discurso está usado en el sentido tradicional de texto organizado en la expresión formal escrita o verbal y no en la forma que tomará más tarde a partir de la obra de Foucault, quien lo consideró como práctica, influyendo decisivamente el uso del término en la posmodernidad.
Se observa la preocupación del autor por la motivación de la expresión, por el sentido interior de las expresiones articuladas de la persona, aspectos accesibles al conocimiento sólo a través de la interpretación. La idea de subtexto fue expresada por primera vez en la psicología soviética por Vygotsky. Esa idea define la imposibilidad de estudiar los fenómenos psíquicos más complejos a través de las expresiones directas de la persona.

La psicología soviética, sin embargo, no especificó ninguna opción ontológica clara sobre los diferentes niveles de la psique, ni consiguió especificar la subjetividad como ontología específica de la psique humana en el contexto de la cultura. Vygotsky, tanto en su obra Psicología del Arte, como en sus trabajos de la última parte de su obra, definida por mí entre 1932 y 1934 (González Rey, 2008), enfatizó el carácter generador de la psique a partir del reconocimiento del impacto de las emociones y de sus consecuencias sobre la vida psíquica, sin ninguna relación inmediata y linear con referentes externos. Sin embargo, nunca pudo especificar cómo la multiplicidad de fenómenos y procesos de la vida social se expresaban en la psique.

Parece ser que el uso de la categoría de sentido en su obra estuviese dirigido a cubrir la ausencia de una unidad psicológica, capaz de dar cuenta de una nueva definición ontológica de los procesos psíquicos desarrollados culturalmente (Leontiev, 1992, 2001; González Rey, 2002; 2004; 2008); sin embargo, como defiendo en trabajo más reciente (González Rey, 2008), creo hoy que eso representó más una hipótesis de trabajo apoyada en el curso de su obra, que una intención real de Vygotsky.

Vygotsky atribuyó un valor a las emociones en el desarrollo de la vida psíquica, que ningún otro psicólogo, soviético, ni extranjero, consiguió desarrollar. Así, en su trabajo Sobre el problema de la psicología del actor creativo (1984), uno de los trabajos de ese último momento de su obra, él escribe: "Las emociones entran en nuevas relaciones con otros elementos de la vida psíquica, nuevos sistemas surgen, nuevos conjuntos defunciones psicológicas; unidades de nivel superior 
emergen, gobernadas por leyes especiales, dependencias mutuas y formas especiales de conexión y movimiento" (p. 328).

En la cita se deja abierta la posibilidad de pensar en un nuevo sistema psíquico que se configura en el proceso de vida del sujeto a partir de las nuevas relaciones de las emociones con otros elementos de la vida psíquica. Vygotsky no se limita a afirmar el carácter generador de lo emocional, lo que ya había hecho en Psicología del Arte, sino que afirma la expresión de todo un sistema en movimiento en que irán apareciendo unidades psíquicas de nivel superior. Entre todas las categorías desarrolladas por él, la que más se aproxima a ese tipo de unidades fue el sentido; sin embargo, el sentido permaneció asociado a la palabra en su definición, lo cual limita la posibilidad de considerarlo como una nueva unidad de la vida psíquica. De cualquier forma, la categoría de sentido abre una posibilidad para pensar la psique en constante desarrollo en las actividades humanas, pues integra la palabra al sistema más general de la psique, la cual está en proceso constante a través del lenguaje.

Sin embargo, Vygotsky no avanzó teóricamente para hacer del sentido un nuevo momento cualitativo de su obra. La consideración del sentido como una nueva unidad de la vida psíquica no podía ser sólo una nueva definición teórica, pues implicaba el cambio de un conjunto de principios más generales sobre los que se organizaba, tanto su obra, como la psicología soviética en aquel momento. El reconocimiento por Vygotsky de la especificidad de las emociones y de su papel activo y generador en la vida psíquica, creó condiciones totalmente nuevas para el desarrollo del tema de la subjetividad en una perspectiva históricocultural; sin embargo, la emergencia de ese tema en las condiciones político-ideológicas de aquel tiempo era completamente imposible.

El propio Vygotsky, en lo que he definido recientemente como segundo momento de su obra (González Rey, 2008) dio un "giro objetivista" al afirmar la interiorización como la génesis de las funciones psíquicas superiores. Ese periodo objetivista fue cercano a los principios sobre los que Leontiev desarrolló la teoría de la actividad, y que fue criticado por Rubinstein (1964) en los siguientes términos:

Toda actividad material externa del hombre contiene ya en su interior componentes psíquicos (fenómenos, procesos) por medio de los cuales se regula. No es lícito, reduciendo la acción del hombre a su mera parte ejecutiva externa, eliminar totalmente de su acción los componentes psíquicos, situando los procesos psíquicos "internos" fuera de la actividad "externa" del hombre, como se hace consciente o inconscientemente, explícita o implícitamente, cuando se afirma que la actividad psíquica surge como resultado de interiorizar la actividad externa (p. 340).

La crítica de Rubinstein destaca el carácter psíquico de la actividad, el que se pierde en la consideración operacional-objetal que define Leontiev. Sin embargo, el término psíquico va resultando demasiado impreciso y genérico para indicar los procesos específicos que forman parte de la acción humana. Creo que el énfasis en la acción humana al analizar la psique, no se puede reducir a los aspectos sensoriales y cognitivos, que también son psíquicos, sino que tiene que considerar el sentido que toma la acción en su ejecución, lo que especifica una cualidad particular de la psique humana: la subjetividad. Pero la definición de sentido aportada por Vygotsky resulta insuficiente para significar este proceso.

Rubinstein va a usar el concepto de vivencia como esa unidad esencial para explicar el carácter subjetivo de la experiencia social de la persona, término que, al igual que el de sentido, Vygotsky también usa en la última parte de su obra, en un esfuerzo fallido por representar la unidad de lo cognitivo y lo afectivo en el desarrollo humano. Es interesante que al igual que Vygotsky, Rubinstein enfatice los aspectos emocionales que forman parte de la vivencia. Para ambos las emociones son esenciales para comprender la especificidad de las formas más avanzadas del psiquismo humano; sin embargo, ante la presión de la idea de reflejo y de la correspondencia de la psique con la realidad, su representación sobre la psique no 
consigue ser coherente en sus trabajos, identificándose en momentos diferentes con lo teórico, lo cognitivo o lo operacional. La idea de la subjetividad, como nivel más complejo de la psique humana desarrollada en las condiciones de la cultura no fue desarrollada teóricamente por ninguno de ellos.

En su momento más audaz, en el libro por el cual fue acusado de idealista, Principios de Psicología General, Rubinstein nos presenta a través del concepto de vivencia, la psique como producción subjetiva, algo semejante a lo que hace Vygotsky en Psicología del Arte y luego en la última parte de sus trabajos. Rubinstein afirma (1967):

La vivencia de la acción que el sujeto lleva a cabo se hace consciente porque ella se remite a las situaciones objetivas por las cuales viene determinada. Sin embargo es bien manifiesto que el número de estas relaciones es, en principio, infinito. Por ello tampoco existe un consciente ilimitado que todo lo abarque. Ninguna vivencia particular se encuentra desligada de una relación; ninguna vivencia se hace consciente definitivamente en todas sus relaciones objetivas, en su relación con todos los aspectos de la existencia, con los cuales está objetivamente unida. Por ello, la conciencia, la conciencia real del individuo concreto, no es jamás conciencia "pura"... Es siempre la unidad de lo que deviene consciente y lo que resta inconsciente, el conjunto de lo consciente y lo inconsciente, de las transiciones mutuas entrelazadas y muchas veces unidas" (p. 24).

En esa cita Rubinstein reconoce lo inconsciente, como también lo había hecho Vygotsky en Psicología del Arte, pero lo reconoce como una actividad inconsciente inseparable del sistema psíquico en su conjunto. La vivencia no es un reflejo, pues no existe en forma de imagen ni de cognición, no es objetal, como muy bien expresa el autor, "se remite a un número de relaciones objetivas por las cuales viene determinada"; sin embargo, no las puede integrar todas, no es reproductiva, sino una expresión generadora del psiquismo humano.
Sin embargo, el autor tampoco resuelve consecuentemente el problema, pues reconociendo ese carácter complejo y no reproductivo de la vivencia, no defiende su especificidad ontológica. Así, en El Desarrollo de la Psicología: principios y métodos, que fue una de sus últimas publicaciones, escribe (1964): “Es cierto, como se ha dicho más arriba, que la actividad material, práctica, es la primaria; y que la actividad teórica, mental, cuya expresión se da únicamente en el plano interno, solo posteriormente se desprende de la primera..." (p. 339). Rubinstein identifica aquí lo mental con lo teórico, al igual que en el momento de su giro objetivista Vygotsky va a identificar lo mental con lo interno, con operaciones externas e internas que primero aparecen en el plano externo. Ambos tienen momentos en que pareciera que se retractan de la osadía de avanzar en la comprensión de la psique como subjetividad al reconocer su no identidad con ningún aspecto objetivo y su carácter generador como sistema.

Ante la imposibilidad de una redefinición de la psique en términos diferentes, a partir de la comprensión de su génesis histórica, social y cultural (principio defendido por ambos autores), el desarrollo de sus visiones epistemológicas de carácter interpretativo no tuvo un desarrollo empírico consecuente, ni en la obra de ellos, ni en la de sus discípulos. Por largo tiempo la hegemonía de la teoría de la actividad en la psicología soviética se acompañó esencialmente de estudios experimentales de los procesos cognitivos, cuyas bases epistemológicas mantenían un carácter esencialmente positivista.

Debido al carácter ideológico atribuido al materialismo en la psicología soviética, esa psicología se orientó en el plano teórico por la subordinación al principio del reflejo, y que en el plano epistemológico destacó el descubrimiento de leyes, término de franca connotación positivista, orientado a destacar el carácter regular y completamente cognoscible de la realidad.

La psicología soviética no pudo desarrollar de forma explícita una alternativa epistemológica posterior a la muerte de sus pioneros, prevaleciendo la línea experimental asociada a la teoría de la 
actividad. Sin embargo, algunos grupos particulares, dirigidos por investigadores que fueron también muy relevantes en aquella psicología, como Bozhovich (1981), Menchinskaya (1959) y Pushkin (1962), entre otros, desarrollaron formas cualitativas de investigación en los campos del aprendizaje, la personalidad y la motivación. En esas investigaciones los procedimientos cualitativos se desarrollaron sin una conciencia epistemológica explícita.

El desarrollo de una definición diferente de la psique humana en las condiciones de la cultura, no fue posible para aquella psicología, a pesar de haber asumido la génesis cultural de la psique. Terminaron siendo preponderantes las posiciones que identificaban la psique con operaciones, actividad, cognición y procesos intelectuales. Los procesos simbólicos y emocionales no fueron comprendidos en su articulación compleja en el curso de la actividad humana, ni fue comprendida la forma en que lo social se organizaba a nivel psicológico. La idea de reflejo no permitió comprender la subjetividad como definición ontológica específica, que no se interioriza, sino que expresa una producción simbólico emocional sobre la experiencia vivida, estrechamente asociada a las emociones que aparecen en la red de experiencias vividas, las que no existen como hechos palpables sino como sistemas de consecuencias inteligibles, sólo a partir de la configuración subjetiva de quienes las viven.

La psicología soviética no organizó teóricamente una representación ontológica diferenciada sobre la psique, lo que implicó que, a pesar de sus indiscutibles avances, no pudiera superar la taxonomía tradicional, en que la psique aparece definida por diferentes tipos de procesos relacionados entre sí, sin un modelo teórico que permita avanzar en la construcción del sistema que articula esos procesos por su sentido psicológico. La psicología soviética, a pesar de su vocación teórica y al papel diferente que consiguió atribuir a la teoría, no pudo trascender la limitación de la psicología empírica de orientarse a la búsqueda de las leyes de su objeto, el cual se constituyó como externo al proceso de producción del conocimiento. En este sentido, las intuiciones epistemológicas de sus pioneros nunca se concretaron en una alternativa epistemológica consistente.

Me gustaría extender este análisis histórico a otras direcciones de la psicología que considero muy importantes y que tienen especial relevancia en el momento actual, como el pospsicoanálisis, la teoría de las representaciones sociales, el constructivismo (en sus posiciones posracionalistas), y el construccionismo social, a las cuales les he dedicado bastante atención en algunas de mis últimas publicaciones (González Rey 1996, 2002, 2004, 2007, 2008), sin embargo, dejo este propósito para otro artículo.

\section{La subjetividad en una perspec- tiva histórico cultural, yendo más allá de la psicología soviética: un análisis ontológico-epistemológico}

En mi trabajo decidí desarrollar uno de los posibles legados de la psicología soviética, que permaneció ignorado en la psicología occidental, e incluso en la propia psicología rusa possoviética; me refiero al tema de la subjetividad en la perspectiva histórico-cultural.

Como he expresado en trabajos anteriores (2004, 2007,2008 ), la subjetividad no fue un tema propio de la modernidad, a pesar de lo afirmado por muchos autores. La modernidad se caracterizó por el cógito y la conciencia y, por alguna razón, como afirma Jameson (2004), esos conceptos se fueron diluyendo en los contornos más generales e inespecíficos de una mención a la subjetividad que nunca encontró una definición específica. La modernidad fue el primado de la racionalidad, las representaciones y las formulaciones operacionales, mientras la subjetividad se orienta a un tipo de producción humana que está más allá de la razón, que es imposible reducir al orden de la representación y que no se puede formalizar en recursos metodológicos precisos, lo que hizo que muchos autores modernos la representaran como elementos de distorsión, como impedimento de la objetividad. En la propia psicología moderna no fue posible especificar lo subjetivo, ni en su génesis, ni en su naturaleza, siendo reducido siempre 
a algo "diferente" de la propia subjetividad: la pulsión, los procesos neurodinámicos, las necesidades, etc.

La subjetividad como producción humana expresa alternativas a las limitaciones objetivas que imponen la realidad y la propia condición humana, como magistralmente expresa Vygotsky (1984), aunque sin mencionar el término, cuando expresa:

Ellos no comprendieron (se refiere a los psicólogos que trabajaban con la defectología en la época) que el "handicap" no representa sólo el empobrecimiento de un estado psicológico, sino una fuente de bienestar, no sólo una debilidad, sino una fortaleza"..." La psicología de la ceguera es esencialmente la psicología de la victoria sobre la ceguera (p. 57).

La subjetividad es un tema congruente en la perspectiva histório-cultural, pues representa un nivel diferenciado de la psique en las condiciones de la cultura, condición necesaria para el propio desarrollo de la cultura. A pesar de los fenómenos diversos que participan en su génesis, sociales, biológicos, históricos, etc., la subjetividad no es la expresión inmediata de ninguno de ellos, sino una producción a partir de formas y procesos simbólicos de naturaleza cultural que son inseparables de las emociones. Las relaciones de una madre con su hijo se vinculan con su temperamento, con la fuerza y urgencia de sus necesidades, con la regularidad o no de sus hábitos, con comportamientos de carácter congénito e innato; sin embargo, ante todo, constituyen un espacio simbólico-emocional de carácter subjetivo y cultural, que será el escenario de la configuración subjetiva de la maternidad para esa madre.

Al partir del concepto de sentido de Vygotsky, y asumiendo las importantes ideas de Rubinstein sobre la vivencia y su carácter siempre incompleto en relación con los múltiples aspectos de la realidad que afectan al hombre, introduje el concepto de sentido subjetivo (González Rey, 1999), en cuya definición he venido avanzando, aunque desde el primer momento definí su especialidad en la unidad de lo simbólico y lo emocional que fundamenta el carácter subjetivo de la experien- cia. En esa unidad lo simbólico evoca lo emocional y viceversa, en un proceso de naturaleza recursiva donde uno evoca la emergencia del otro, sin convertirse nunca en su causa (González Rey, 2002). La categoría en sentido subjetivo expresa el carácter generador de las emociones humanas, presente tanto en Vygotsky como en Rubinstein, tema profundamente ignorado por la psicología desde sus posiciones racionalistas dominantes.

La negación del carácter generador inconsciente de las emociones, como destacan tanto Vygotsky como Rubinstein, expresó una visión determinista que no permitía comprender la génesis de las emociones en los efectos colaterales de las relaciones y eventos vividos. Las emociones son una consecuencia subjetiva de esos eventos, y no como su reproducción. Las emociones tienen en su base la configuración subjetiva actual de quien las expresa. Las experiencias, aisladas de la organización subjetiva de quien las vive, no tienen ninguna significación. Los efectos colaterales de lo vivido se expresan sobre todo en la imaginación y la fantasía, ambas de una naturaleza emocional irreducible a cualquier evento objetivo.

Es precisamente esa expresión generadora de la psique humana la que se representa en los sentidos subjetivos. Los sentidos subjetivos definen el aspecto subjetivo de la experiencia vivida y existen no como contenidos puntuales, susceptibles de expresión concreta por el sujeto, sino como conjunto de emociones y procesos simbólicos que se articulan alrededor de definiciones culturales sobre las que se desarrolla la existencia humana, tales como madre, padre, hermano, sexo, inteligencia, moral, patriotismo, religión. Todas esas dimensiones simbólicas de las prácticas humanas aparecen como sentidos subjetivos y configuraciones subjetivas que se ínter penetran entre sí en el curso de la experiencia generando continuamente nuevos procesos subjetivos que escapan al control racional, y a la intencionalidad del sujeto. La sociedad se organiza en representaciones sociales que naturalizan y objetivan sus propias producciones subjetivas, aquellas que permiten sus prácticas compartidas, y le hacen sentir a los participantes que su acción se apoya en una "verdad" de carácter objetivo. Sobre esa base se orientan 
todas las formas de fanatismo, así como algunas de las creencias más consolidadas de la llamada sociedad "democrática", como por ejemplo, la "verdad" desmoronada, ante la incredulidad de muchos, de que el mercado se autorregula, olvidando que el mercado está formado por relaciones entre personas y que su dimensión subjetiva está más allá de todos los saberes y recursos técnicos humanos. A nombre de esas "verdades" como patria, religión, ideología, etc., se han cometido las mayores barbaries de la humanidad.

La subjetividad es una dimensión que incomoda, pues elimina las ideas de control, de racionalidad, de futuro, de certeza, de objetividad y de normalidad, en la forma en que ellas aparecieron en el pensamiento moderno. La subjetividad destituye los absolutos a los que se han querido subordinar históricamente las prácticas humanas, incluyendo la reducción de todo fenómeno humano a la condición de práctica discursiva. La reificación de lo discursivo es una forma de recreación de lo racional, pues al suponer la subordinación de la persona al orden discursivo, se ignora la multitud de efectos diferentes que la realidad genera sobre los sujetos y los escenarios de las prácticas discursivas, las que no representan un flujo simbólico abstracto.

Al presentar la subjetividad como opción de inteligibilidad de los fenómenos humanos, no la reifico, no intento un reduccionismo subjetivo de esos fenómenos, sino que aspiro solamente a convertirla en una dimensión necesaria para el estudio de las diversas prácticas y procesos humanos de institucionalización, pues históricamente la subjetividad ha quedado fuera de los análisis de las ciencias sociales, entre las cuales, la que más ha trabajado esa dimensión ha sido la sociología (Ferraroti, 2003 y Touraine, 1998, entre otros).

Al defender la subjetividad como definición ontológica intento separarme de su substancialización en ciertos tipos de cuestiones y contenidos. No la veo ni como individual, ni como intrapsíquica, ni como lo íntimo sino como una definición presente en todos los procesos y niveles de las producciones humanas, desde las individuales hasta las institucionales, expresándose en cualquier tipo de producción social. A partir de esto defiendo la existencia inseparable de la subjetividad social e individual, estando cada una de ellas presente en la otra, pero no como algo externo a ella, sino como momento de sentido subjetivo de esa otra producción (González Rey, 1991).

La motivación humana es central en la idea de subjetividad. Los sentidos subjetivos son unidades motivacionales, están en la base de las emociones que alimentan toda práctica y proceso humano. La motivación es una compleja red de elementos, irreducible a un contenido concreto; sin embargo, el concepto de motivación fue víctima, así como el de cognición y de otros procesos psíquicos, del reduccionismo elementalista sufrido por la psicología; la motivación no es, ni puede ser, un elemento o una tendencia concreta, la motivación es la expresión de un sistema, la subjetividad, en la configuración subjetiva de todo acto humano. Todo acto evoca y produce una configuración de sentidos subjetivos que es su base dinámica y que se expresa de diferentes formas en la propia procesualidad del acto.

La subjetividad en esta propuesta integra la procesualidad de la acción, es inseparable de ella. Las configuraciones subjetivas no representan un "a priori" que determina la acción; ellas son parte del sentido subjetivo que una acción tiene desde su propio comienzo; sin embargo, ellas no "dirigen" los sentidos subjetivos que se van desarrollando en la acción: ellas son un momento inseparable en la producción de esos sentidos subjetivos.

Como muy bien ha destacado Mitjans (2007), a quien debo esa precisión, los sentidos subjetivos existen en la procesualidad de las acciones y relaciones. En ocasiones, en la propia evolución de estos conceptos, las definiciones de sentido subjetivo y configuración subjetiva se han confundido, mezclado; una configuración subjetiva es una organización relativamente estable de sentidos subjetivos relacionados con un evento, actividad o producción social determinados. Ella es parte inseparable de los sentidos subjetivos que aparecen en el proceso de acción y de las relaciones que ocurren en cualquier espacio de la vida, y po- 
demos acceder a esas configuraciones subjetivas sólo a través de los sentidos subjetivos diferenciados que se generan en la acción, los que están siempre referidos a las configuraciones subjetivas implicadas en la acción y que especifican al sujeto de esa acción.

Los sentidos subjetivos, a diferencia de las categorías asociadas a los referentes estáticos universales que por mucho tiempo fueron hegemónicas en la psicología, tienen las siguientes características:

- No son operacionalizables, no pueden ser traducidos en elementos concretos estandarizados, pues ellos son de naturaleza simbólicoemocional y están en proceso permanente; ellos toman formas diferentes en el comportamiento y sólo pueden ser construidos por las interpretaciones del investigador. Los sentidos subjetivos no se pueden definir a priori, para desde esa definición orientar un análisis sobre un material; ellos nacen en el propio proceso de análisis y construcción de un material empírico. Esto ha estado en la base de nuestro énfasis en el carácter constructivointerpretativo del conocimiento.

- No existen conjuntos de sentidos subjetivos "listos" para ser conocidos. Sentido subjetivo de realización, de prestigio social no existen, reconocerlos de esa forma sería importar la lógica extensiva, estática, y fragmentada de la psicología, a esta nueva definición; autoestima, realización, interés, etc., son manifestaciones simbólico-emocionales particulares, que se expresan en una multiplicidad de sentidos subjetivos diferentes. Un sentido subjetivo siempre integra diversas fuentes emocionales y sus desdoblamientos simbólicos.

- En las diferentes actividades humanas coexisten sentidos subjetivos que expresan emociones y procesos simbólicos contradictorios. Los sentidos subjetivos no se regulan ni por la intención, ni por la razón, son verdaderas producciones, subjetivas. Ellos se configuran en el curso de la vida de la persona.

- La categoría de sentido subjetivo rompe con la visión determinista de la psique, pues los sentidos subjetivos son producciones generadas a partir de las configuraciones subjetivas implicadas en la acción, ellos no aparecen de forma directa por el carácter de una experiencia vivida. Las configuraciones subjetivas no excluyen al hombre como sujeto de la acción, pero son parte de las vivencias que aparecen en esa acción, lo que, de hecho, las coloca más allá de cualquier control intencional del sujeto.

- La relación de los sentidos subjetivos con la realidad en que la persona vive no es lineal, ni inmediata. Los sentidos subjetivos son el resultado de efectos colaterales de la vida social, en cuya emergencia las configuraciones subjetivas actuales del sujeto y de sus diferentes espacios sociales de acción, representan el escenario de organización subjetiva de esos efectos. Los efectos colaterales son artefactos de relación, no influencias externas concretas. De esta forma, los sentidos subjetivos y sus configuraciones nos informan de manera indirecta y poco ordenada sobre los diferentes ámbitos sociales de la persona.

\section{Implicaciones epistemológicas del reconocimiento ontológico de los sentidos subjetivos y sus configu- raciones para la psicología}

El estudio de los sentidos subjetivos y sus configuraciones se inscribe en una idea de ciencia orientada a la producción de modelos teóricos, la que tiene diferencias esenciales con una ciencia inductivo-deductiva de carácter empírico. La ciencia que se apoya en modelos teóricos no aspira a demostraciones empíricas sino a la creación de opciones de inteligibilidad sobre el problema que estudia. Lo empírico no representa la vía por la cual se llega a un postulado conceptual; el modelo es la vía que permite el acceso a lo empírico. La mecánica cuántica inauguró una ciencia apoyada en modelos teóricos y, aunque no explotó ese concepto teóricamente, representó la primera ciencia en ponerlo en práctica. Así: la idea de modelo aparece de forma nítida en la siguiente afirmación de Heisenberg (1995):

... objeto de conocimiento científico jamás es conocido directamente de la observación, 
esto es, de la experimentación, pero sí por la construcción teórica (o postulado axiomático), especulativamente propuesta, y evaluada indirecta y experimentalmente por las consecuencias que son deducidas de aquella construcción" (pp. 12).

Ese énfasis en que el conocimiento no es una expresión directa de la realidad, paradójicamente, no entró en el imaginario de las ciencias sociales hasta muy tarde. Bourdieu, Chamboredon \& Passeron se aproximan a la idea de modelo cuando escriben:

... un sistema de hipótesis contiene su valor epistemológico en la coherencia que constituye su plena vulnerabilidad; por una parte un solo hecho puede cuestionarlo integralmente, y, por la otra, construido a costa de una ruptura con las apariencias fenomenales, no puede recibir la confirmación inmediata y fácil que proporcionarían los hechos, tomados en su valor superficial o los documentos en forma literal (1975, p. 92).

En nuestra definición sobre la Epistemología Cualitativa (González Rey, 1996), ya había afirmado el carácter constructivo de la producción del conocimiento. Introduje ese término para explicitar las características del tipo de investigación cualitativa que me proponía desarrollar, la que era esencialmente diferente de los abordajes que enfatizaban el carácter descriptivo e inductivo de ese tipo de investigación (Glasser \& Strauss, 1967; Bogdan \& Taylor, 1975, Lincoln \& Guba, 1985, entre otros).

La Epistemología cualitativa que se apoya en la definición del carácter constructivo-interpretativo del conocimiento, defiende entre otros de sus principios epistemológicos esenciales la significación de lo singular en la producción del conocimiento científico y el carácter dialógico de la investigación psicológica. El valor del uso del caso singular está estrechamente asociado a la idea de modelo, a pesar de que al definir la Epistemología Cualitativa, todavía no había desarrollado esa idea.

El valor de lo singular está dado por la información relevante que su estudio aporta al modelo en de- sarrollo en el proceso de investigación; sólo dentro de ese modelo las informaciones singulares van a adquirir un sentido. Un modelo está representando por un conjunto de ideas e hipótesis relacionadas entre sí, que apoyadas en un marco teórico, representan una fuente de inteligibilidad en que convergen las informaciones resultantes de los diferentes instrumentos y situaciones de la investigación con las ideas del investigador, que avanzan como hipótesis en proceso en ese modelo.

El modelo teórico es una herramienta de acceso a sistemas complejos y diferentes de información que, gracias al modelo, permiten el desarrollo de nuevos indicadores sobre la información empírica que adquieren significado sólo dentro del modelo en cuestión. El modelo representa una construcción teórica con capacidad de desarrollo en el momento empírico y que se expresa en el desarrollo progresivo de hipótesis y construcciones del investigador.

Así, por ejemplo, en el estudio de una persona que siente depresión, y que se atribuye por ella al exceso de carga de trabajo, un investigador orientado por nuestro marco teórico sobre la subjetividad, de entrada, desecha que esa sea la razón de la depresión, simplemente porque todo estado psicológico o comportamiento, desde esta perspectiva, tiene en su base una configuración de sentidos subjetivos, irreducible a un elemento único. Cuando el estudio de caso sobre esa persona avanza, comienzan a aparecer indicadores que apuntan a posibles sentidos subjetivos que podrían estar implicados en esa depresión.

Así, en este ejemplo, la persona estudiada expresa lo siguiente en el completamiento de frases:

-Mi esposo es muy inmaduro y gasta dinero sin control.

-Con mi experiencia actual creo que nunca me habría casado.

-Mis momentos más felices: cuando converso con mi hijo y puedo compartir mi tiempo de forma descontraida con él.

- Me preocupa la seguridad económica de la familia en el futuro. 
-Quisiera que mi esposo volviera a encontrar trabajo.

Las informaciones anteriores obtenidas del completamiento de frases, permiten abrir una hipótesis importante sobre la cual la persona no habló, y sobre la cual comenzaría el desarrollo de un modelo teórico orientado al conocimiento de la configuración subjetiva que aparece en esa depresión. Ese modelo no agota la configuración subjetiva de la depresión, pero abre un camino de investigación que será legítima en tanto permita inteligibilidad sobre nuevas informaciones provenientes del sujeto. Es sobre la base de esa inteligibilidad que se mantendrá la viabilidad del modelo en el curso de la investigación.

Las frases anteriores permiten abrir la hipótesis de que sus relaciones con el marido representan un conflicto, cuyas expresiones y efectos indirectos podrían estar asociados al referido estado depresivo. Esa hipótesis se apoya en los siguientes aspectos del completamiento de frases:

Al marido lo identifica como inmaduro y gastador lo que, unido a la preocupación por la situación económica futura de la familia, representa una combinación que permite suponer, de forma indirecta, rechazo por su marido, idea que gana fuerza cuando, de forma explícita, afirma que, con su experiencia actual no se hubiera casado nunca. Por otra parte, el hecho de mencionar sus alegrías y momentos positivos sólo en relación a su hijo, representa otro indicador que reafirma el valor heurístico de la hipótesis formulada sobre el conflicto o rechazo al marido. El desarrollo de esa hipótesis, y su integración con otras ideas y reflexiones producidas en el análisis del material representan el curso del modelo teórico que, en este caso, está orientado a la definición de la configuración subjetiva de la depresión. Sea este estudio un momento de una investigación, o un caso de diagnóstico, el modelo creado para acompañarlo podría conducir al desarrollo de otro modelo más complejo, iniciando el camino más ambicioso de estudiar el desarrollo de las configuraciones subjetivas de la depresión, lo que representaría una línea de investigación.
Esta perspectiva de investigación cualitativa, ilustrada en múltiples de mis investigaciones, y de otras desarrolladas por colegas de nuestro grupo de trabajo, implica la participación activa y permanente del investigador en el desarrollo de un modelo teórico que va a crecer sólo a partir de sus construcciones teóricas sobre los indicadores que fundamentan la viabilidad de aquellas en el momento empírico. Debo recordar que viabilidad no es demostración, sino el desarrollo de nuevas construcciones que reafirman las anteriores o las contradicen, pero conservando el mismo corpus teórico en desarrollo. El proceso de investigación desde esta perspectiva constructivo-interpretativa, se apoya en el desarrollo hipotético de un modelo teórico que no se alimenta de afirmaciones absolutas apoyadas en los resultados, sino en la convergencia de múltiples resultados empíricos con los núcleos de significación desarrollados en el curso del modelo.

Desde esta perspectiva el dato no existe fuera de un sistema de ideas o de una representación teórica; ningún elemento empírico representa un significado fuera de un sistema de significados, por lo cual, los aspectos cuantitativos asociados a mediciones o correlaciones estadísticas no representan recursos conclusivos, sino momentos de un proceso de significación. El número deja de representar un resultado, siendo sólo un nuevo momento de significación dentro de un proceso que sólo se define como sistema, y no por resultados aislados tomados en relación.

Esta forma de trabajar la construcción de la información permite visualizar conceptos teóricos que, como los sentidos subjetivos, no tiene una expresión fija, ni pueden ser captados directamente de las manifestaciones concretas de las personas. Uno de los mayores desafíos del estudio de la subjetividad en esta perspectiva, es que entre las representaciones conscientes susceptibles de expresión verbal intencional por la persona y las configuraciones subjetivas que están en la base de los estados y conflictos referidos en esas representaciones, no existe ninguna relación directa, por lo que las hipótesis sobre esas configuraciones sólo pueden ser desarrolladas por vía 
indirecta a partir de hipótesis sobre sistemas de información abiertos asociados a las más diversas expresiones de las personas. Es a partir de aquí que definí el instrumento de pesquisa como todo aquel recurso o actividad desplegada por el investigador que facilita la expresión emocionalmente comprometida de la persona (González Rey, 1996).

Esta forma de investigación representa una aproximación metodológica nueva, apoyada en principios epistemológicos diferentes a los que tradicionalmente han hegemonizado las formas tradicionales de investigación cualitativa.

La psicología está hoy en un momento importante de redefiniciones y de desarrollo de nuevas alternativas teórico-epistemológicas, que tendrán importantes repercusiones para el desarrollo de la metodología de investigación. Sin embargo, es importante enfatizar que las metodologías no son procedimientos en abstracto, sino procesos que guardan una estrecha relación con aquellas definiciones teóricas que las sustentan, representando las vías de producción y significación de información empírica susceptible de apoyar y extender el curso de esas definiciones teóricas en el campo de la investigación psicológica. De ahí el énfasis que en este artículo he puesto frente a los aspectos ontológicos y epistemológicos en la investigación psicológica.

\section{Algunas reflexiones finales}

El desarrollo de la psicología se ha visto afectado, quizás como el de ninguna otra ciencia social, por la base epistemológica empírica de sus investigaciones que, durante gran parte del siglo pasado, hegemonizó la idea de investigación científica. Muchos problemas importantes para el desarrollo de la psicología fueron ignorados por ese tipo de investigación dominante. Ese cuadro, muy bien analizado en la obra de autores como Koch. S. (1981) y Danziger, K. (1990) en la psicología, llevó a un culto al método científico que influyó mucho en la ausencia de discusión sobre las cuestiones ontológicas y epistemológicas de la psicología. El purismo cientificista de la psicología también in- fluyó en su separación de la filosofía y de otras ciencias sociales.

El psicoanálisis, a pesar de representar una alternativa epistemológica diferente, no consiguió explicitar de forma teórica consistente los principios nuevos que apoyaron su producción de conocimiento en la práctica clínica, lo que le impidió defender esa práctica en lo que ella representaba como opción epistemológica diferente. Como he defendido en el presente artículo, la ausencia de conciencia epistemológica en el psicoanálisis no le permitió el perfeccionamiento de los recursos para producir conocimiento a partir de lo empírico y legitimarlo en una perspectiva interpretativa de carácter hermenéutico.

La ausencia de un referente teórico en la psicología académica, capaz de expresar las complejidades del hombre en las condiciones de su existencia social y de abordar los aspectos subjetivos de las acciones humanas en diferentes campos, llevó sobre todo a la sociología y a la antropología, a tomar al psicoanálisis como referencia para las cuestiones subjetivas.

La subjetividad sólo puede ser comprendida por una teoría que considere su génesis social y cultural; esa relación aparece por primera vez con la psicología soviética, que crea las condiciones para el desarrollo del tema desde una perspectiva completamente diferente. A pesar de los avances que autores como Vygotsky y Rubinstein conquistaron en esa dirección, en general, ni ellos, ni las orientaciones principales de aquella psicología, consiguieron defender el tema de la subjetividad en su especificidad ontológica y epistemológica.

Este trabajo cierra con la posición que he venido defendiendo sobre la subjetividad en una perspectiva histórico-cultural, la cual, debido a su carácter complejo y a su desconocimiento en la historia de la psicología, genera un conjunto de demandas epistemológicas y metodológicas que me llevaron a poner un énfasis en el carácter constructivo-interpretativo de la ciencia, representado por la idea de modelo teórico y por la atribución de un nuevo lugar para la teoría en la investigación psicológica. 


\section{Referencias}

Bogdan, R. \& Taylor, S.D. (1975). Introduction to Qualitative Research Methods: A Phenomenological Approach to the Social Sciences. New York: Wiley.

Bourdieu, P., Chamboredon, J.C. \& Passeron, J. (1975). El oficio del psicólogo. México: Siglo XXI.

Bozhovich, L.I. (1981). La personalidad y su desarrollo en la edad infantil. La Habana: Pueblo y Educación.

Danziger, K. (1990). Constructing the subject: historical origins of psychological research. New York: Cambridge University Press.

Ferraroti, F. (2003). On the science of uncertainty. New York: Lexington Books.

Gadamer, H. (2007). Hermenêutica em retrospectiva. A virada hermeneútica. Vol II. Petrópolis: VOZES.

Glasser, B. \& Strauss, A. (1967). Discovery of Grounded Theory: strategies for qualitative research. Chicago: Aldine.

González Rey, F. (1991). Subjetividad, sujeto y psicología social. Conferencia magistral. Congreso Interamericano de Psicología. San José de Costa Rica.

González Rey, F. (1996). Epistemología cualitativa y subjetividad. La Habana, Cuba: Pueblo y Educación. (Publicado también por la EDUC. San Pablo. 1997).

González Rey, F. (2002). Sujeto y subjetividad: una aproximación histórico-cultural. México. D.F.: Thomson.

González Rey, F. (2004). O social na psicología e a psicologia social: a emergência do sujeito. Petrópolis. Brasil: VOZES.
González Rey, F. (2007). Social and individual subjectivity from an historical cultural standpoint. En Critical Social studies. Outlines. 9 (2), pp. 3-14.

González Rey, F. (2008). Different periods in Vygotsky's work: their implications for arguments regarding his legacy. Personal presentation in the Annual meeting of the International Society for Cultural and Activity Research. San Diego. California.

Heisenberg, W. (1995). Física \& Filosofía. Brasilia: UNB.

Jameson, F. (2004). Una modernidad singular. Ensayo sobre la ontología del presente. Barcelona: Gedisa.

Jones. E. (1920). Editorial. International Journal of Psychoanalysis, 1 (1).

Koch, S. (1981). The nature and limits of psychological knowledge: lesson of a century of qua "science". En American Psychologist, 3 (36), pp. 257-269.

Leontiev, A. A. (1992). Ecce Homo. Methodological Problems of the Activity theoretical approach. En Multidisciplinary Newsletter For Activity Theory, 1 (12), pp. 41-45.

Leontiev, A. A. (2001) Deyatelnii Um (Deyatelnosti, snack, lichnosti) La Mente Activa (Actividad, signo, personalidad. Moscú: Smysl.

Lincoln, Y.S. \& Guba, E.G. (1985). Naturalistic inquiri. Beverly Hills: Sage Publications.

Menchinskaya, N. A. (1959). Psychology of the mastery of knowledge in school. Moscow: APN Press.

Mitjans A. (2007). Reunión grupo de investigación sobre subjetividad. Brasilia.

Pushkin, V.N. (1962). Vopr psikhol, 8, p.156.

Roudinesco, E. (1995). Genealogías. Río de Janeiro: Relume Dumara. 
Rubinstein, S.L. (1964). El desarrollo de la psicología: principios y métodos. La Habana: Editora del Consejo Nacional de Universidades.

Rubinstein, S.L. (1967). Principios de Psicología General. La Habana: Editora Revolucionaria.

Touraine, A. (1998). Poderemos viver juntos. Petrópolis: VOZES.

Vygotsky, L.S. (1965). Psykjologiya Iskustva (Psicología del Arte) Izdatelstva Iskustva. Moscú: Editorial Arte.
Vygotsky, L.S. (1984). K voprocy o psykjologii judochestvennovo tvorschestva aktera (Sobre la cuestión de la creatividad artística del actor) Sobranie Sochineniya. Vol. 6. Moscú: Pedagógica.

Vygotsky, L.S. (1987). Thinking and Speech. In The collected works of L.S.Vygotsky Vol. 1 (Eds. Rieber, R \& Carton, A.), pp 43-287. New York: Plenum Press.

Vygotsky, L.S. (1993). Defect and Compensation. In The collected works of L.S.Vygotsky. Vol. 2 (Eds. Rieber, R. \& Carton, A.), pp 52-64. New York: Plenum Press. 